\title{
Unsteady Aerodynamics of an Oscillating Fastback Model
}

\author{
Author, co-author (Joshua Baden Fuller, Martin A Passmore)
}

Affiliation (Loughborough University)

\begin{abstract}
This paper investigates the surface pressures found on the sides of a Davis model under steady state conditions and during yawed oscillations at a reduced frequency which would generally be assumed to give a quasi-static response. The surface pressures are used to investigate the flow field and integrated to infer aerodynamic loads. The results show hysteresis in the oscillating model's results, most strongly in the A-pillar flows. The changes to the flow field reduce strength of the flows around the rear pillars, reduce the strength and extent of the A-pillar vortex and cause the surface pressures to couple with the oscillating motion. This work shows the flows around the front of a vehicle may be more important to a vehicle's unsteady aerodynamics than is generally accepted and also leads to the conclusions that the reduced frequency parameter may not fully describe the onset unsteadiness.
\end{abstract}

\section{INTRODUCTION}

The majority of vehicle aerodynamic development is based on time averaged aerodynamic coefficients measured using a static vehicle or model in highly uniform onset flows. In practice, even under nominally steady state conditions, instantaneous results show that the flow fields around fastback, notchback and squareback shapes are highly unsteady (Bearman [1], Gilhome et al [2], Duell and George [3] among others) with the flow fields containing periodic shedding, base pumping, flow field unsteadiness and a number of other features. However, steady onset conditions are rarely experienced in normal driving, where the onset flow is often highly unsteady due to a wide range of influences including vehicle speed and direction, the presence of other traffic, road side topography and varying weather conditions.

One form of unsteady aerodynamic input that has received significant research attention is the effect of naturally occurring crosswinds and extreme crosswind gusts. These have been investigated using a range of methods, including oscillating models and onset winds, crosswind gust generators and models that move across a windtunnel (eg Chadwick et al [4], Ryan and Dominy [5] Garry and Cooper [6], Mansor and Passmore [7], Theissen et al [8]). These methods have produced inconsistent results, in some case showing aerodynamic coefficients measured under transient conditions to be larger than those measured on a static model and in others the same or smaller. Within these results is evidence of flow field hysteresis, Guilmineau and Chometon [9], and hysteresis has also been found in the lift and pitching moment coefficients recording during pitching and heaving oscillations by Aschwanden et al [10] and also Nakashima et al [11]. Unsteady crosswind tests also show periodic features within the flow field altering due to the unsteady onset wind, Schröck et al [12] and different response times from different flow structures around the vehicle, [5].

This paper adds to the existing body of work by investigating the surface pressures on a fastback model in an unsteady onset flow created by oscillating the model in the yaw plane.

\section{EXPERIMENTAL METHOD}

The experiments were carried out in the $1 / 4$ scale windtunnel at Loughborough University. It has a 
closed working section and fixed floor and is arranged in an unusual ' $U$ ' shape. The working section has a maximum speed of $45 \mathrm{~m} / \mathrm{s}$ with baseline turbulence intensity of 0.2 and flow uniformity of $\pm 0.4 \%$; for more details see Johl et al [13].

A Davis model, figure 1 , sized to approximately $1 / 6$ of full scale was used in these experiments. It has a $20^{\circ}$ backlight angle and all the edges are rounded with a $20 \mathrm{~mm}$ radius. The model was made of a light weight fibre glass shell and was mounted $40 \mathrm{~mm}$ from the windtunnel floor with a single $\varnothing 20 \mathrm{~mm}$ steel shaft through the centre of the model.

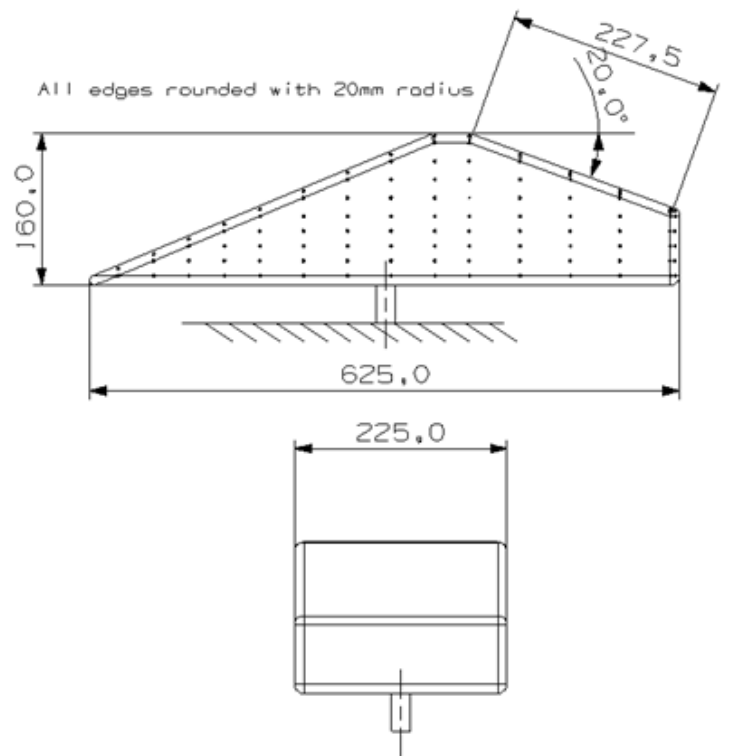

Figure 1, Davis model, showing principle dimensions and pressure tapping locations

The model becomes Reynolds number insensitive in drag coefficient at $\mathrm{Re}=1.3 \times 10^{6}$ based on overall length and all the results in this paper were collected at $40 \mathrm{~m} / \mathrm{s}$ giving a Reynolds number of $1.7 \times 10^{6}$. The backlight angle of $20^{\circ}$ creates an attached flow down the backlight and creates a typical trailing vortex flow structure in its wake. There is a thorough investigation of the flow field around this model at $0^{\circ}$ yaw reported in Fuller and Passmore [14].

Each side of the model was covered in 84 pressure tappings, shown in figure 1 and pressure data was collected under steady state and oscillating conditions.

The pressures were recorded using two 64 channel miniature pressure scanners accurate to $\pm 0.15 \mathrm{~mm}$ $\mathrm{H}_{2} 0$. Each pressure tapping was sampled 8192 times, triggered by an externally supplied $260 \mathrm{~Hz}$ signal, representing 31 seconds of data. Distortion caused by the tubing linking the model surface to the pressure scanner was removed using an experimentally derived correction function found using a similar method to that used by SimsWilliams and Dominy [15]. The time base of the sampling was also corrected by re-sampling so that at each time step all the data is concurrent rather than sequential, from tapping 1 to 64 . The surface pressures are presented as pressure coefficients based on freestream dynamic pressure and are corrected for blockage using a first order approximation of applying the MIRA blockage correction to the pressure coefficient equation, where $e$ is the ratio of the model frontal area to the work section area.

$$
C_{p}=\frac{C_{p}+2 e}{(1+2 e)}
$$

In the steady state tests the model was mounted to the underfloor turntable and yawed in steps of $1^{\circ}$ in the range $\pm 12^{\circ}$. At each yaw angle a settling time was allowed before pressure data was collected.

A sinusoidal oscillating yaw motion was produced by attaching the model support shaft to a crank driven by an electric motor, illustrated in figure 2 . The support beam was bolted under the wind tunnel floor and the model was supported with two bearings on the shaft.

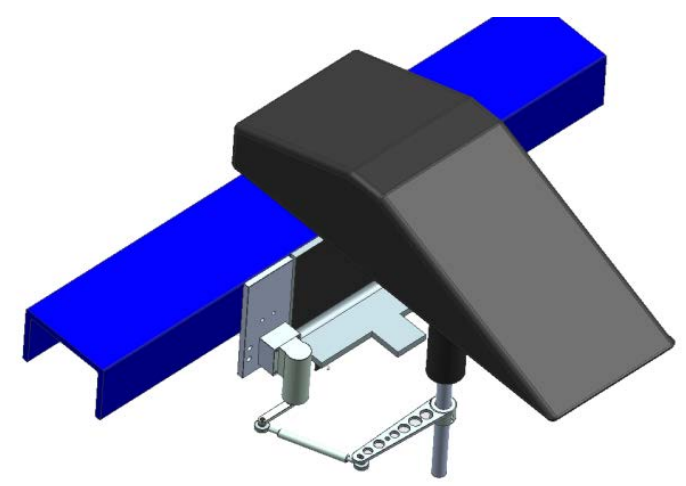

Figure 2. The motored crank system that drives the oscillating model motion.

The driven crank produced a consistent and repeatable $1 \mathrm{~Hz}$ oscillation with yaw angle amplitude of $\pm 11^{\circ}$, which, when combined with a test speed of $40 \mathrm{~m} / \mathrm{s}$, produces a reduced frequency, as defined by Sims-Williams [16] in equation 1, of 0.098 , meaning the motion falls within the quasistatic approximation region of reduced frequency $<$ 0.1 . 


$$
K=\frac{2 \pi f l}{u}
$$

This oscillating motion was based on the work of Mansor [7] who used a sprung oscillating rig with the same Davis model. He found that the model produced a self-excited yaw oscillation at low reduced frequencies with amplitude around $11^{\circ}$ which could not be explained using quasi-static approximations despite the low reduced frequency.

The motion was subject to slight cycle to cycle variations in the frequency, $\pm 0.02 \mathrm{~Hz}$, and amplitude, $\pm 0.2^{\circ}$, about the mean values. These were caused by small, low frequency changes to the supply voltage and unsteadiness in the aerodynamic forces acting against the motor.

As in the steady state tests, the pressures were collected in batches of 8192 samples collected at an externally triggered $260 \mathrm{~Hz}$. This was repeated 33 times without stopping between the data sets; in total 2Gbs of data was collected. As the model oscillated, the instantaneous yaw angle, which was measured using an angular potentiometer mounted to the model support shaft, and the external trigger signal were sampled at $2 \mathrm{kHz}$ using a simple data acquisition program. This data was used to find the yaw angles at which the pressure data was sampled. The pressure data was processed to recreate a mean unsteady surface pressure for an average oscillation. Having corrected for the tubing distortion and time base, the data from each tapping was split up into sections 512 samples long starting as the model was at $0^{\circ}$ yaw with an increasing yaw angle; this produced just over 1000 smaller data sets. Using Matlab, the Fourier transform of each smaller data set was found and these were ensemble averaged before being converted back into the time domain. This creates a mean surface pressure history at each pressure tapping during a cycle of motion. Using this method to process the data removes the small effects of the frequency and amplitude variations from the signal in way that 'binning' or taking a simple arithmetic mean of the pressures would not. Due to the cycle to cycle unsteadiness of the model motion, these methods would produce incorrect results as a consequence of averaging values that are not created by the same amplitude or frequency of motion.

Both the steady state and oscillating model surface pressures are used to plot contours over the side of the model and are also integrated over their surrounding area to infer side force and yaw moment coefficients.

\section{RESULTS AND DISCUSSION}

Using the surface pressures to calculate the area weighted yaw moment coefficient, the steady state results, shown by crosses in figure 3 , produce near linear results with a positive gradient. The results are slightly offset from $0^{\circ}$; this is caused by slight differences in the model geometry on each side and small differences between the tapping locations on each side of the model. This is particularly bad on curved surfaces with large pressure gradients, differences of even $1 \mathrm{~mm}$ in the relative positions of the pressure tappings can make a significant difference to the side to side pressures.

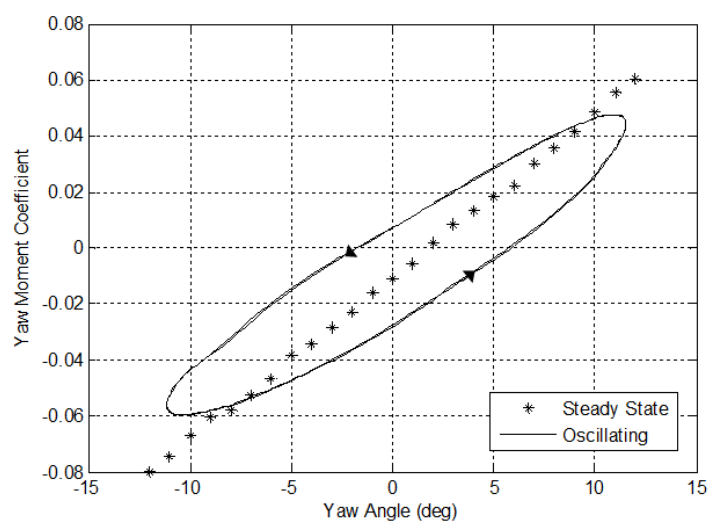

Figure 3. Area weighted yaw moment coefficients calculated from surface pressures

Appendix A contains a full set of contour plots showing how the pressures change on the side of the model as it is yawed. The changes are summarised in figure 4 that shows the absolute difference in surface pressure on the two sides of the model at $-10^{\circ}$ yaw. On the leeside of the model the A-pillar vortex, which dominates the side force, is strengthened and on the windward side the flow into the trailing vortex and around the vertical rear edge is increased. Together these create a positive yaw moment gradient.

The pressure plots are slightly 'steppy' and this is an unfortunate and unavailable consequence of the spacing between the tappings and the interpolation used between them. 



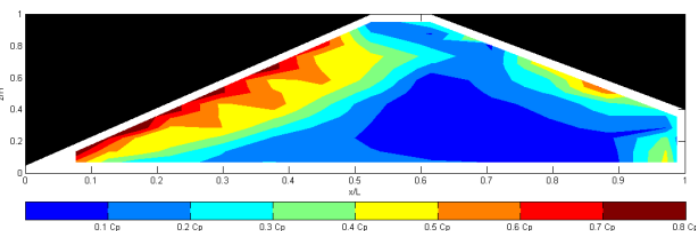

Figure 4. The absolute difference between the surface pressures on the two sides of the model at $-10^{\circ}$ yaw.

As well as the steady state results, figure 3 also shows the yaw moments calculated from the surface pressures on the oscillating model. The results clearly show that the yaw moment on the oscillating model contains hysteresis, similar to that found on the Willy model in [9]. The mean gradient of the oscillating model results has reduced compared to the steady state results and this disagrees with [9] which was centred on the steady state results. However, whether this is result is real or a consequence of the maxima and minima of the surface pressures moving relative to the pressure tappings is not known.

The contributions to the overall oscillating model side force from the front and rear of the model are presented in figure 5 .

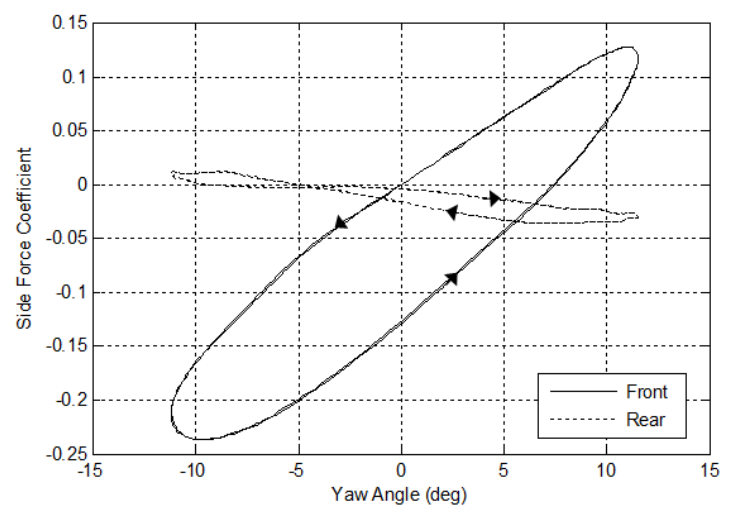

Figure 5. Area weighted side force coefficients.

In agreement with figure 4 the largest contributor to overall yaw moment and its hysteresis is from the front half of the model, especially from the region under the A-pillar vortex. There is a much smaller contribution from the rear and this is in agreement with the positive yaw moment gradient seen in figure 4.

Whilst most road vehicles have a positive yaw moment gradient and large contributions from the front of the car, the large amount of hysteresis in the front side force is not a commonly seen result.
Theissen et al [8] and Wojciak et al [18], working with an oscillating scale model of an executive saloon, only found transient effects towards the rear of the models and in their wakes. This general result, where the unsteady effects are at the rear of the model or vehicle, is commonly reported, further examples include [9] and [12].

While the importance of the A-pillar vortex and the hysteresis in the front side force is possibly partially model specific, with the Davis model having an unusually long front which extends for $55 \%$ of the model length, there is a small but growing amount of work that indicates the importance of the front of the model to the aerodynamic unsteadiness. Using the same model as used in this paper, mounted downstream of a pair of oscillating aerofoils, Passmore et al [17] found the A-pillar region was dominated by an amplitude reduction on the unsteady model with an increasing phase lag along the model.

Oettle et al [19] used CFD to simulate unsteady onset flow yaw angles on a Jaguar XF and found hysteresis in the A-pillar flows towards the top of the front window, the differences between steady state and transient were greatest on the leeside. The importance of the front of the vehicles was also reported by Tsubokura et al [20] who found that differences in the vortex emanating from the front wheel arch of a generic saloon car manifested themselves as surface pressure differences on the side of the vehicle behind the rear wheels.

The hysteresis in the lateral aerodynamic coefficients goes against the predicted response based on the low reduced frequency but is in agreement with Mansor's self-exciting response. However, despite proposing the boundaries for different responses based on the reduced frequency, Sims-Williams [16] also questions the usefulness of the current form of the reduced frequency equation and suggests that an amplitude term may need to be included to better describe the unsteady test conditions.

There is a side by side comparison of the surface pressures on the static and oscillating models in Appendix A that qualitatively shows the differences between the surface pressures on the two models. The sources of the side force on the oscillating model are the same as on the static model and the differences between the two model types can be summarised as a reduction in the 
intensity of the A-pillar and rear pillar flows and a phase lag in the oscillating model pressures.

Further understanding of the changes to the flow structures can be achieved by comparing the quasistatic and oscillating surface pressures at individual pressure tappings. The quasi-static signal is generated by arranging the steady state values in a time series to simulate a yaw angle oscillation. This takes into account the higher yaw rates at small yaw angles and the slower yaw rates at larger yaw angles. Figure 6 shows a comparison taken from under the A-pillar and figure 7 shows a comparison taken from the edge of the roof.

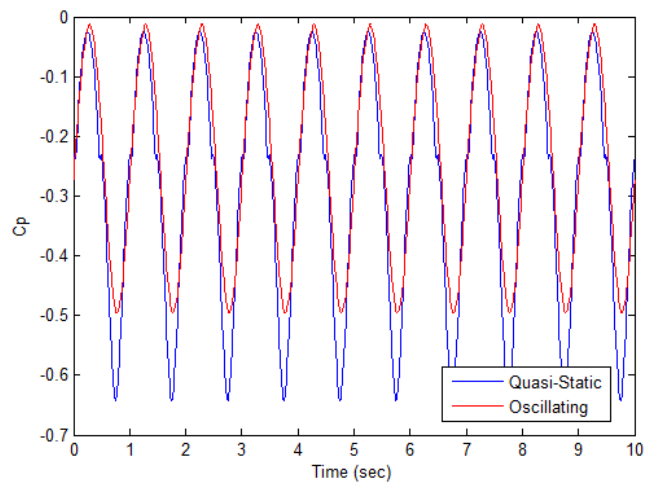

Fgure 6. Comparison of quasi-static and oscillating model surface pressures under the Apillar

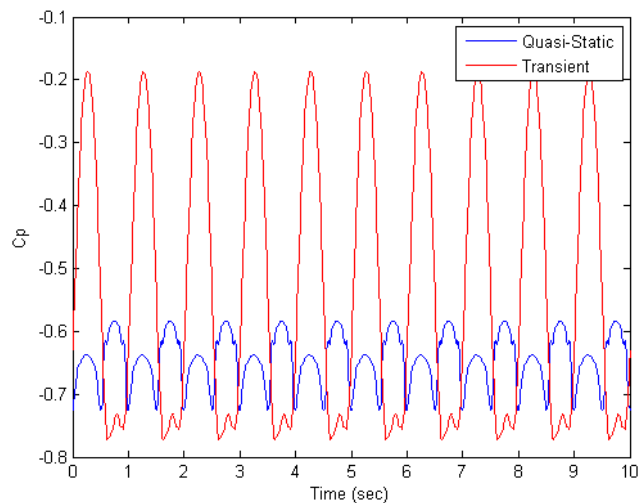

Figure 7. Comparison of quasi-static and oscillating model surface pressures from the start of the roof

Figure 6 shows a slight change in the phase and amplitude between the two pressure signals but they have the same dominant frequency of $1 \mathrm{~Hz}$ that matches the model motion. Figure 7 shows a much more complex change: the dominant frequency of the signal is $2 \mathrm{~Hz}$ in the quasi-static signal and $1 \mathrm{~Hz}$, coupled with the model motion, in the oscillating model signal. Associated with this change is a large increase in the amplitude of the signal.
There are two interesting points that arise from this figure; the quasi-static signal is twice the motion frequency and the oscillating model motion causes the surface pressures to couple with the motion.

The result in figure 7 is a function of the A-pillar vortex and the flow into the windward trailing vortex. Under steady state conditions, the leeside A-pillar vortex extends to the top $1 / 3$ of the trailing pillar and on the windward side of the model this region experiences comparably low surface pressures due to the flow into the trailing vortices creating a dominant quasi-static surface pressure frequency in this region of the model of $2 \mathrm{~Hz}$. The leeside A-pillar vortex on the oscillating model is weaker and does not extend as far over the side of the model as on the static model. The pressure tappings in the region are then only affected by either the A-pillar vortex or the flows into the trailing vortices, rather than by both flow structures. This changes the dominant frequency from $2 \mathrm{~Hz}$ to $1 \mathrm{~Hz}$, coupling the unsteady surface pressures to the model motion, and significantly altering the range of surface pressures by inherently changing the flow structures present.

Further investigation into this frequency change shows that these changes are found across a large region of the centre and rear of the model. This can be split into two with different flow features driving the changes in each section; the approximate extents of the areas affected are shown in figure 8.

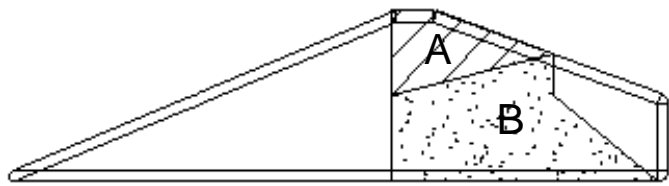

Figure 8, regions of changes to the dominant frequencies

The upper region, $\mathrm{A}$, is created by the leeside Apillar and windward rear pillar flows as explained. The lower region, $\mathrm{B}$, is created by the high pressure that moves back and forth along the side of the model as it yaws. There is a slight difference between how this moves along the model on the quasi-static and oscillating models which changes the dominant frequency from $2 \mathrm{~Hz}$ on the quasistatic model to $1 \mathrm{~Hz}$. This is a much subtler effect than higher up the model but nonetheless remains 
an interesting result showing the coupling between model motion and surface pressures.

Although this frequency coupling is interesting, comparing figure 8 to figure 4 shows that the areas of the model affected by this frequency change only make a small contribution to the overall aerodynamic loads acting on the model; hence this frequency change is actually not significant to the overall aerodynamics loads acting on the model. This is in agreement with McCroskey [22] who stated that during dynamic stall on aerofoils the individual surface pressures showed a much greater change from steady state to oscillating results than the subsequent aerodynamic loads.

Figure 9 shows the phase and amplitude relationship between the quasi-static and oscillating model pressure signals. The regions highlighted in figure 8 should be ignored, as it is incorrect to compare the phase and amplitude of signals with different dominant frequencies.
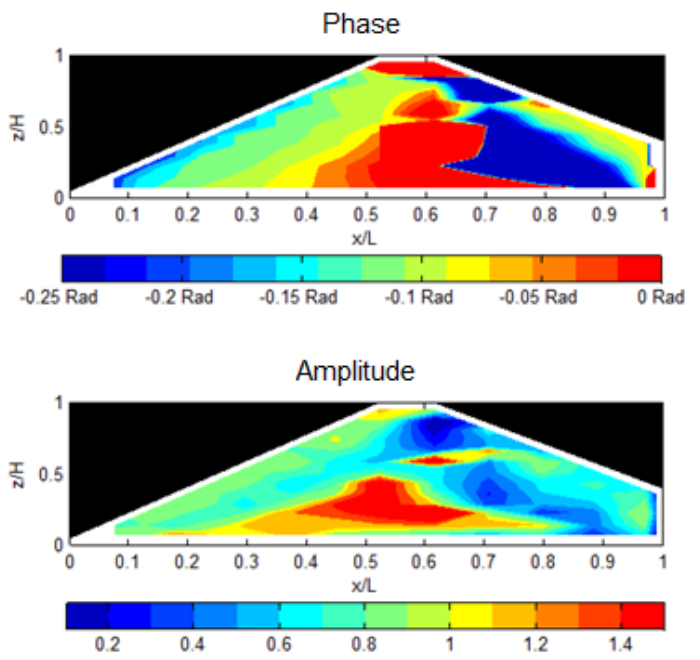

Figure 9, phase and magnitude changes to the surface pressures

Under the A-pillar vortex there is a phase lag that is largest on the leading edge of the A-pillar and reduces further along and lower down the model, indicating the A-pillar hysteresis is not driven by the pure lag term. Along the A-pillar edge the gains are very close to one but below this the oscillating model surface pressure gains reduce to a minimum of 0.7 along the A-pillar vortex reattachment line. Lower down and towards the centre of the model the amplitude of the surface pressure changes in the centre of the model is larger than under quasi-static conditions. The centre and rear half of the model is dominated by the changes in the dominant frequencies but on the rear pillar the frequencies do match. Here the gains are below one and the phase results show a lag compared to the quasi-static results. However, the proximity of the fully separated flow on the vertical rear edge of the model and the unusual results in the centre of the model mean this interpretation is only based on the results of a few pressure tappings.

The results on the front half of the model agree with those reported by Passmore et al [17]. The lag at the rear of the model was also reported in [17] and also on a more realistic geometry in Theissen et al [8] and Wojciak et al [18].

It is likely that the hysteresis in the flow field around the Davis model can be explained based on the causes of dynamic stall on a pitching aerofoil as explained by Ericsson and Reding [21]. Dynamic stall is created by a combination of two effects caused by the model's motion and how this modifies the speed of onset flow over the model's surfaces. There is a pure lag effect which is a fundamental feature of the length and motion of the model and there is a flow field modification term that produces different flow fields around the transient models than around the static models.

The pure lag term is a consequence of the time taken for the onset flow to travel the length of the model. As the model is oscillating the flow at the front of the model is always changing creating a lag as this travels down the length of the model. A similar effect to this has been reported by Passmore et al [17] and also Wojciak et al [18] and it is likely that this is one of the main influences on the flows into the trailing vortices and at the rear of the models but not the A-pillar flows.

The large changes to the A-pillar vortices are likely caused by flow field modification effects of the model motion. The rotation of the model means that on parts of the model, the surface is travelling in the same direction as the flow over that surface. As the yaw angle is increasing away from $0^{\circ}$ the model's leeside surface moves in the same direction as the flow into the leeside A-pillar vortex. Although the surface velocity is only small, at most only $0.06 \mathrm{~m} / \mathrm{s}$, as the model passes through $0^{\circ}$ at the corners of the model where the distance to the axis is the greatest, it modifies the boundary layer and reduces the relative onset flow velocity on the leeside of the model. The reduction in the relative flow velocity reduces the severity of the surface pressure gradient around the A-pillar, enabling the 
flow to remain attached slightly further around the A-pillar than on the static model. The boundary layer modification also reduces separation as the model motion re-energises the boundary layer delaying separation until further around the A-pillar; this effect is analogous to adding blowing in to the boundary layer. When the model is moving back towards $0^{\circ}$ yaw the effects are reversed as the model's leeside surface is moving into the onset flow. This increases the relative velocity, steepening the pressure gradient and promoting earlier boundary layer separation meaning the Apillar vortex separates earlier. These effects will both alter the magnitude of the pressure changes and also the phase of the pressure changes relative to the model motion. The flow modification effects also occur on the windward side of the model, but with the direction of the model surface velocity reversed. However, the windward A-pillar flows are attached and less affected by the transient effects. This can be seen in Appendix A where the largest differences between the steady state and oscillating flows are found on the leeside of the model. This matches a finding by Ryan and Dominy [5] who reported that the leeside flows had a greater delay than those on the windward side of the model. Oettle et al [19] also report that the greatest differences between a transient and steady state pressure under the A-pillar vortex were on the leeside.

There are likely two further causes of the slower response on the model's leeside. Due to the rotation of the model the onset flow reaches the windward side of the model before the leeside creating a very slightly faster reaction in the windward flow structures. Secondly, the windward side of the model is moved positively into the onset flow and the flow field is forced to react by the model being moved into the onset flow. However, on the leeside of the model, a void is created which the flow can move into but the flow field response is less rapid due to the inertia and viscosity of the air.

The reduction in the amplitude of the surface pressures below the A-pillar vortex is from a combination of the modified pressure field upstream of the A-pillar and the slower response time mean that the leeside A-pillar vortex on the oscillating model is less intense than on the static model.

It is possible that the differences between the oscillating and static models may also be created by the effects of freestream turbulence. Newnham et al [23] showed increasing small scale freestream turbulence reduced the critical Reynolds number around a radiused leading edge and promoted an attached flow, such as seen in these results on the leeside A-pillar. Relative to the model coordinates, the onset flow and crosswind flow speed is constantly changing, creating a longitudinal turbulence intensity of 0.0004 and a lateral turbulence intensity of 0.04 . However, these values are extremely low and below the baseline freestream turbulence intensity present within the windtunnel, furthermore the turbulent length scales created by the oscillating motion are significantly larger than the theoretical limit proposed by Newnham that should interact with the shear layers, hence this explanation is not suitable in this situation.

\section{CONCLUSIONS}

This paper investigates the surface pressures on a static and oscillating Davis model and shows significant differences from the expected quasistatic response.

The results do not fit with the predicted response based on the low reduced frequency showing that this parameter does not accurately describe this unsteady test condition.

Although there is hysteresis in both the front and rear side forces, the largest effect is in the front side force. This is in agreement with other recent research into these effects, demonstrating that frontal flows are worthy of further investigation. This result may be particularly relevant for the increasing numbers of 1-box vehicles with continuous bonnet edges and A-pillars.

The surface pressures on the oscillating model couple to with the model motion, changing some of the dominant frequencies from those found using a quasi-static approximation, although this has little effect on the overall loads on the model.

\section{ACKNOWLEDGEMENTS}

The authors would like to acknowledge the excellent technical assistance provided by Rob 
Hunter and Stacey Prentice. We would like to thank Daniel Wood for the considerable time and effort taken to develop the pressure tubing correction function and acknowledge Jeff Howell's valuable advice and guidance.

\section{REFERENCES}

1. Bearman, P W., Near wake flows behind two- and three-dimensional bluff bodies. JWEIA 69-71, 1997, p33-54

2. Gilhome, B R., Saunders, J W., Sheridan, J. Time Averaged and Unsteady NearWake Analysis of Cars. SAE Technical Paper Series, 2001, 2001-01-1040

3. Duell, E G, George, A R, Experimental Study of a Ground Vehicle Body Unsteady Near Wake. SAE Technical paper Series, 1999, 1999-01-0812

4. Chadwick, A., Garry, K., Howell, J., Transient Aerodynamic Characteristics of Simple Vehicle Shapes by the Measurement of Surface Pressures. SAE Technical Paper Series, 2001, 2001-010876

5. Ryan, A., Dominy, R.G., The Aerodynamic Forces Induced on a Passenger Vehicle in Response to a Transient Cross-Wind Gust at a Relative Incidence of $30^{\circ}$. SAE Technical Paper Series, 1998, 980392

6. Garry, K P., Cooper, K R., Comparison of Quasi-Static and Dynamic Wind Tunnel Measurements on Simplified Tractor Trailer Models. JWEIA 22, 186, p185-194

7. Mansor, S., Passmore, M.A., Estimation of bluff body transient aerodynamics using an oscillating model rig. JWEIA 96, 2008, p1218-1231

8. Theissen, P., Wojciak, J., Heuler, K., Demuth, R., Indinger, T., Adams, N., Experimental Investigation of Unsteady vehicle Aerodynamics under TimeDependent Flow Conditions Part 1. SAE Technical Paper Series, 2011, 2011-010177

9. Guilmineau E., Chometon, F. Numerical and Experimental Analysis of Unsteady Separated Flow behind an Oscillating Car
Model. SAE Technical Paper Series, 2008, 2008-01-0738

10. Aschwanden, P., Müller, J.,Knörnschild, U. Experimental Study on the Influence of Model Motion on the Aerodynamic Performance of a Race Car. SAE Technical Paper Series, 2006, 2006-010803

11. Nakashima, T., Tsubokura, M., Nouzawa, T., Nakamura,T., Ichimiya, M. Flow Structures above the Truck Deck of Sedan-Type Vehicles and Their Influence on High-Speed Vehicle Stability. $2^{\text {nd }}$ Report; Numerical Investigation on Simplified Vehicle Models using Large Eddy Simulation. SAE Technical Paper Series, 2009, 2009-01-0006

12. Schröck D; Widdecke, N; Weidemann, J, 2009, Aerodynamic Response of a Vehicle Model to Turbulent Wind. 7th FKFS Conference, Progress in Vehicle Aerodynamics and Thermal Management, October, 2009, Stuttgart Germany.

13. Johl, G., Passmore, M., Render, P. Design Methodology and performance of an indraft wind tunnel. The Aeronautical Journal, 2004, vol 108. Pages 465-473

14. Fuller, J. Passmore M.A., The importance of rear pillar radius on wake structures and unsteadiness. Submitted to JWEIA, March 2012.

15. Sims-Williams, D.B., Dominy, R. G. Experimental investigation into Unsteadiness and Instability in Passenger Car Aerodynamics. SAE Technical Paper Series, 1998, 980391

16. Sims-Williams, D. Cross Winds and Transients: Reality, Simulation and Effects. SAE Technical Paper Series, 2011, 2011-01-0172

17. Passmore, M.A., Richardson, S., Imam, A. An Experimental Study of Unsteady Aerodynamics. IMechE Part D, 215, Feb 2001, p 779-7789

18. Wojciak, J, Theissen, P., Heuler, K., Indinger, T., Adams, N., Demuth, R. Experimental Investigation of Unsteady vehicle Aerodynamics under TimeDependent Flow Conditions Part 2. SAE Technical Paper Series, 2011, 2011-010164 
19. Oettle, N., Mankowski, O., Sims-Williams, D. B., Dominy, R., Freeman, C., Gaylard, A. Assessment of a Vehicle's Transient Aerodynamic Response. SAE Technical Paper Series, 2012, 2012-01-0449

20. Tsubokura, M.m Ikawa, Y., Nakashima, T., Okada, Y., Kamioda, T., Nouzawa, T. Unsteady Vehicle Aerodynamics during a Dynamic Steering Action: $2^{\text {nd }}$ Report, Numerical Analysis. SAE Technical paper Series, 2012, 2012-01-0448

21. Ericsson, L.E., Reding, J.P., Fluid Mechanics of dynamic Stall Part 1.
Unsteady Flow Concepts. Journal of Fluids and Structures, 1988, 2, p1-33

22. McCroskey, W. J., Unsteady Airfoils. Annual review of Fluid Mechanics, 1982, vol14, pages 285-311.

23. Newnham, P. Passmore, M., Howell, J., Baxendale, A., On the Optimisation of Road Vehicle Leading Edge Radius in Varying Levels of Freestream Turbulence. SAE Technical Paper Series, 2006, 200601-1029 
Appendix A

Yaw

$0^{\circ}$

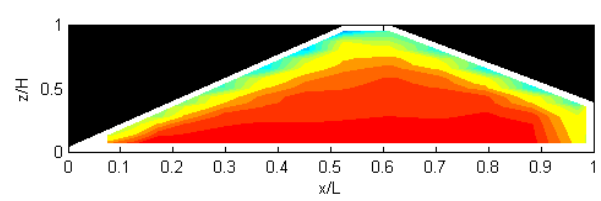

$1^{\circ}$

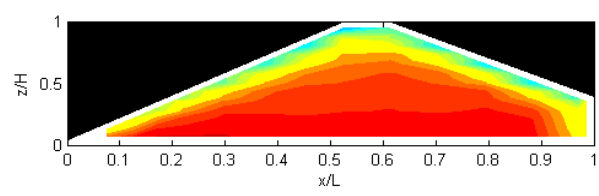

$2^{\circ}$

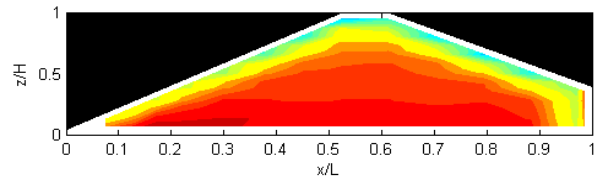

$3^{\circ}$

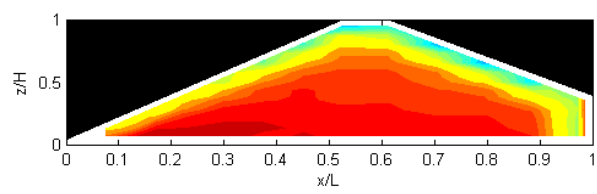

$4^{\circ}$

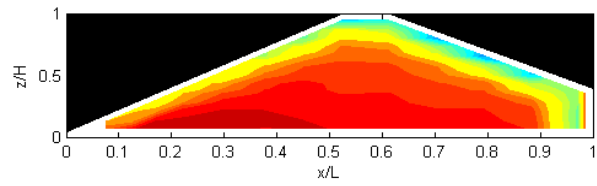

$5^{\circ}$

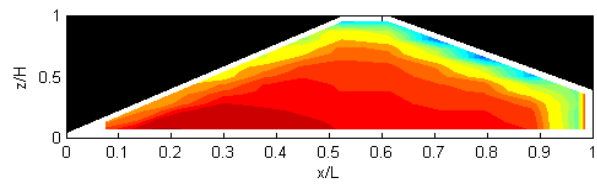

$6^{\circ}$

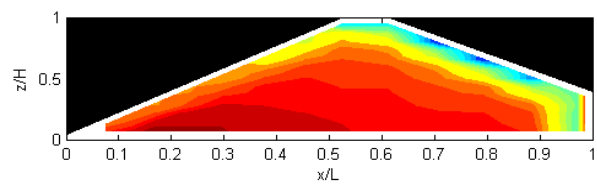

$7^{\circ}$

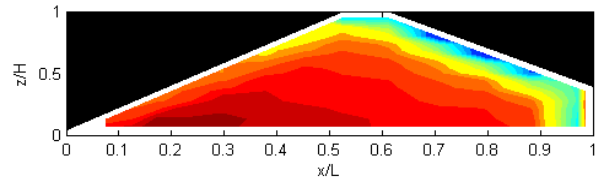

$8^{\circ}$

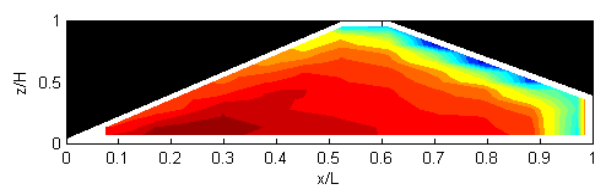

$9^{\circ}$

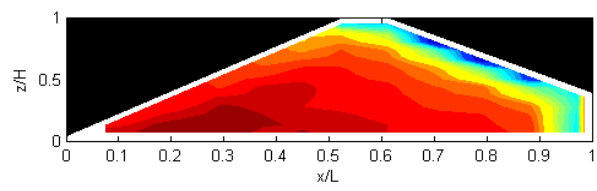

$10^{\circ}$

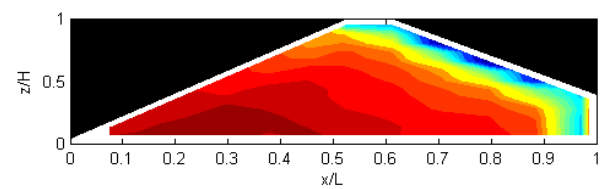

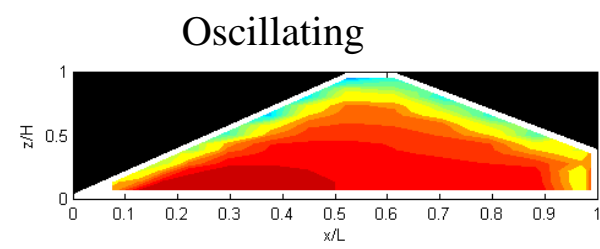
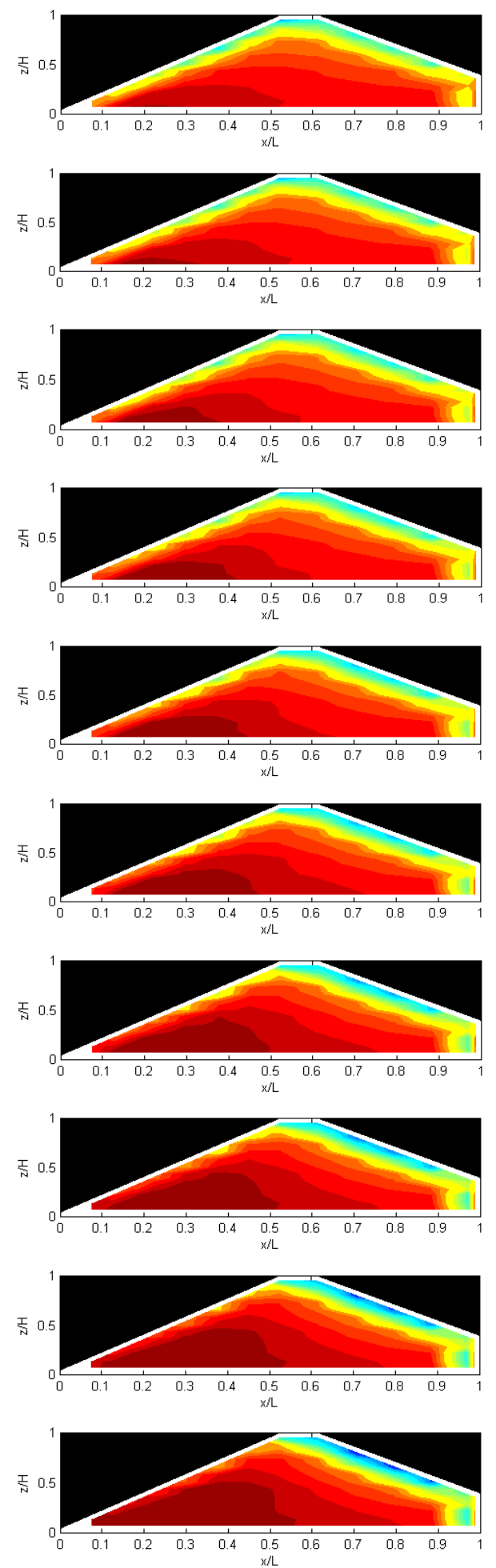
$11^{\circ}$

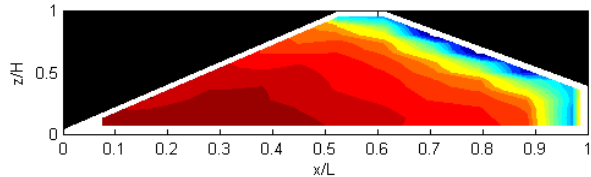

$11^{\circ}$

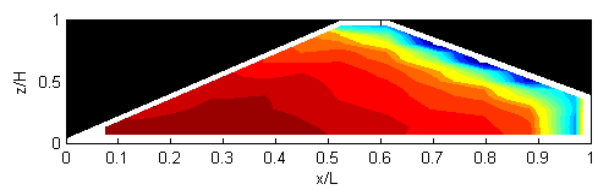

$10^{\circ}$

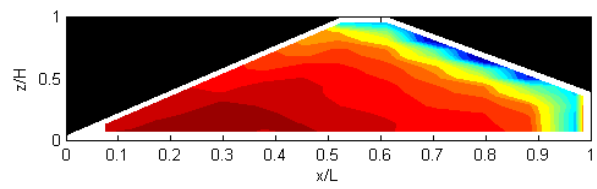

$9^{\circ}$

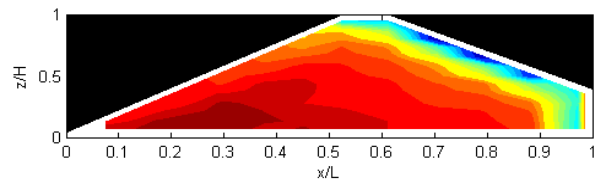

$8^{\circ}$

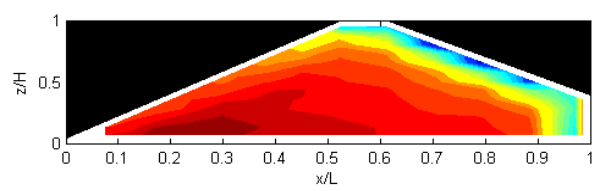

$7^{\circ}$

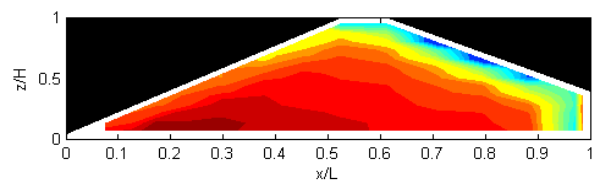

$6^{0}$

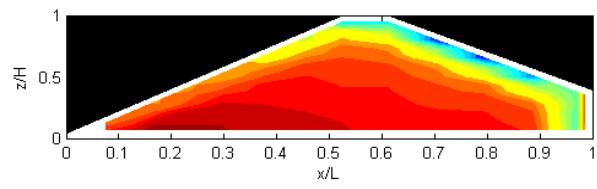

$5^{\circ}$

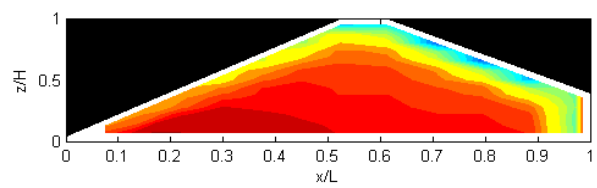

$4^{\circ}$

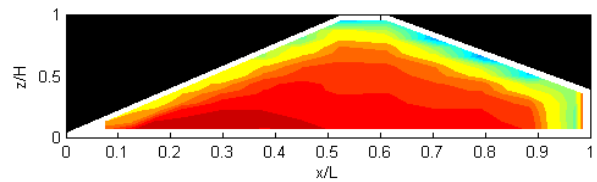

$3^{\circ}$

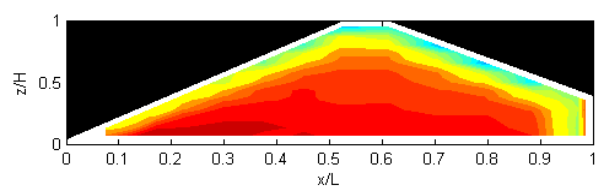

$2^{\circ}$

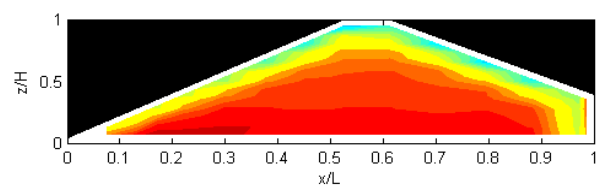

$1^{\circ}$

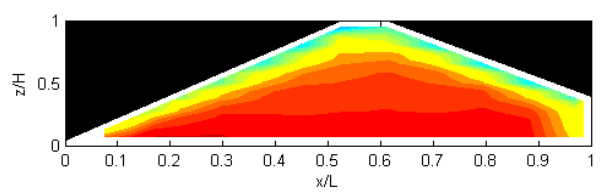

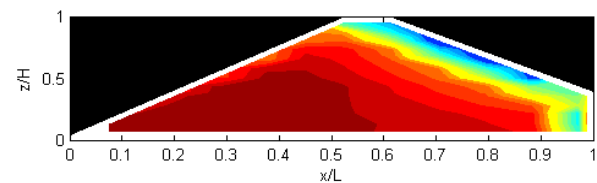
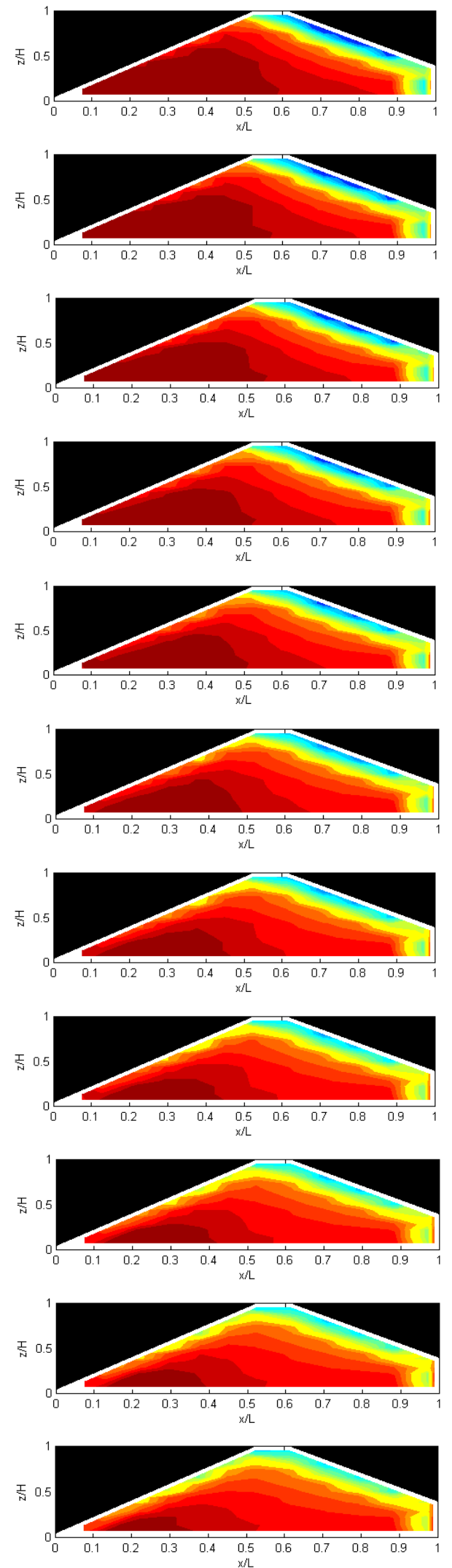

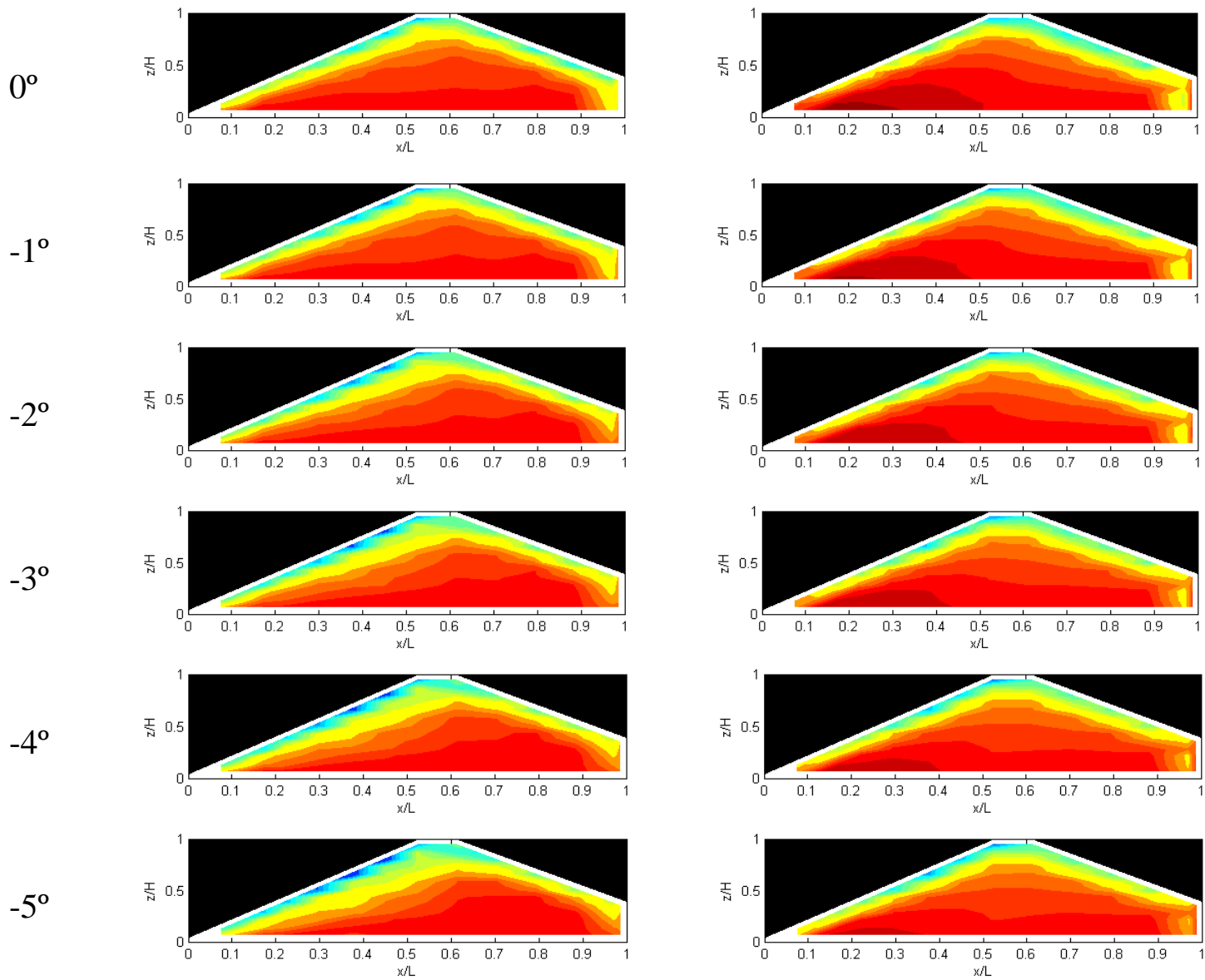

$-6^{\circ}$
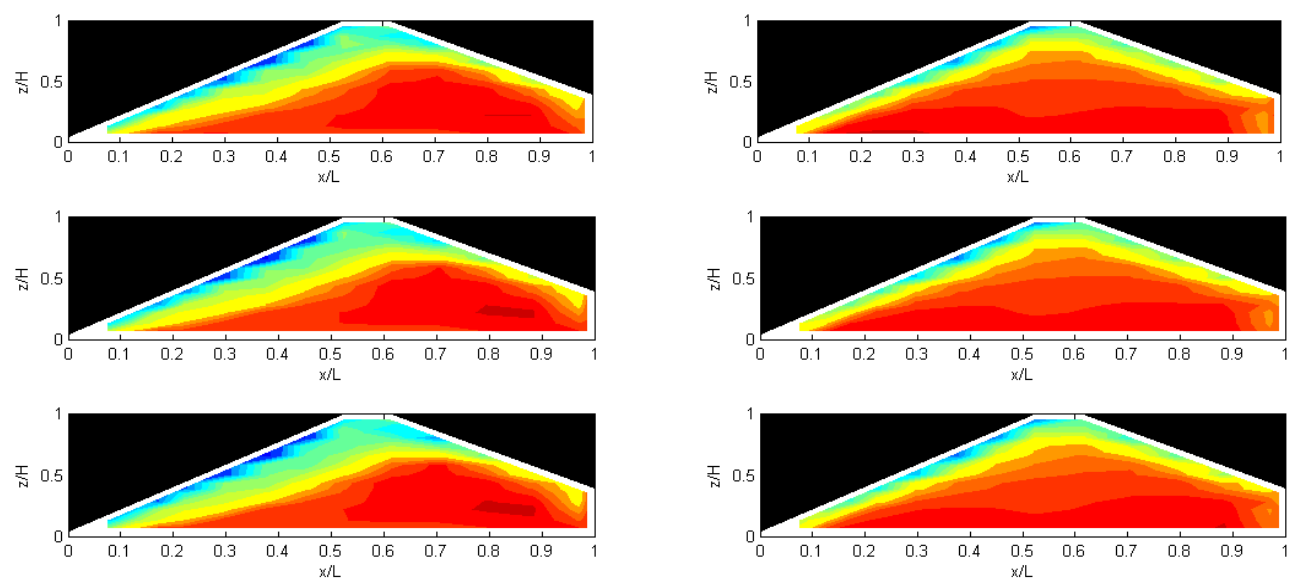

$-9^{\circ}$
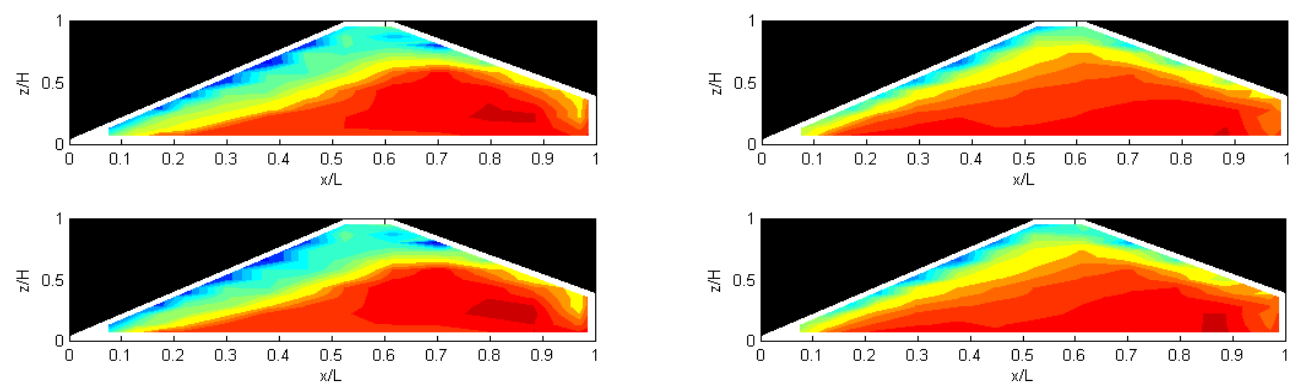

$-11^{\circ}$
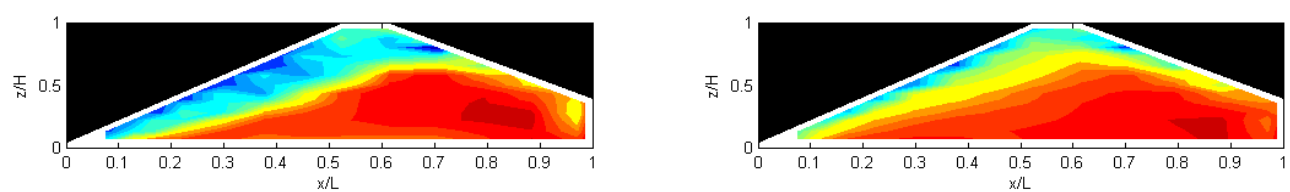
$-11^{\circ}$

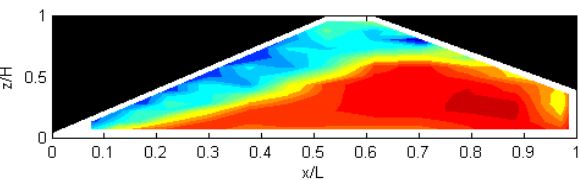

$-10^{\circ}$

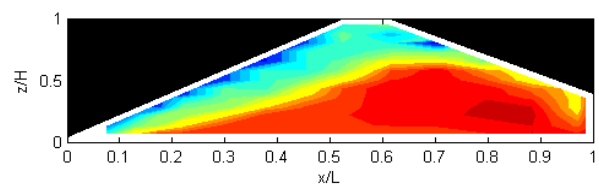

$-9^{\circ}$

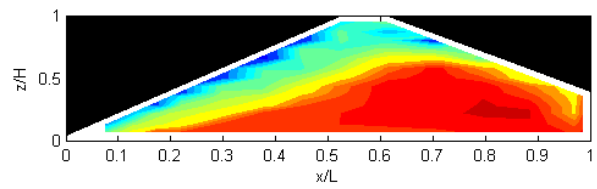

$-8^{\circ}$

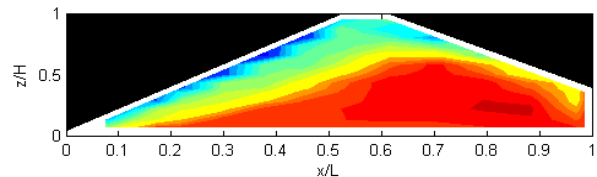

$-7^{0}$

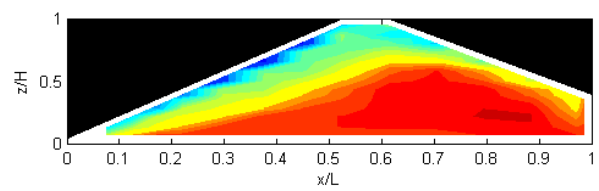

$-6^{\circ}$

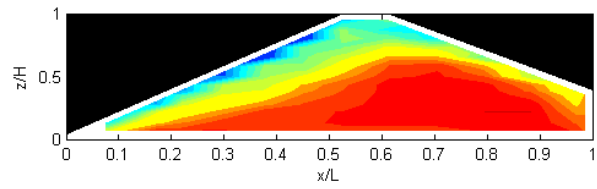

$-5^{\circ}$

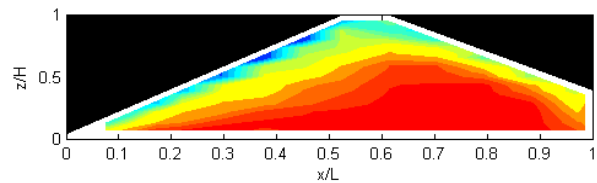

$-4^{0}$

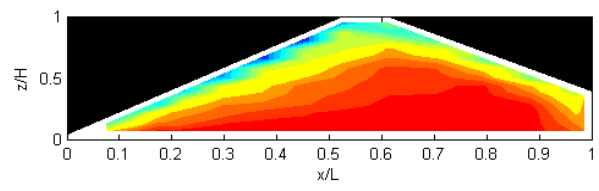

$-3^{\circ}$

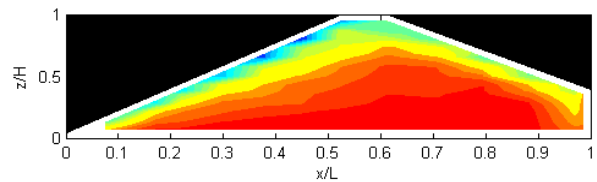

$-2^{\circ}$

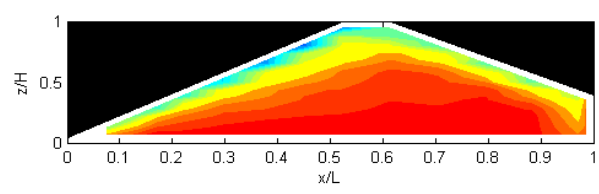

$-1^{0}$

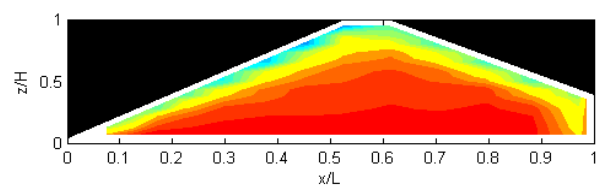

$0^{\circ}$

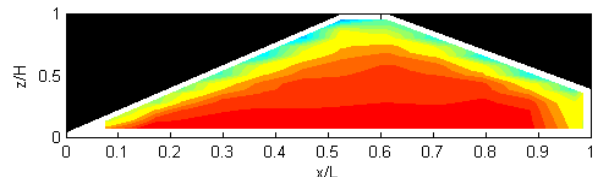

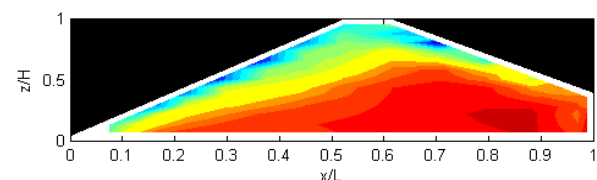
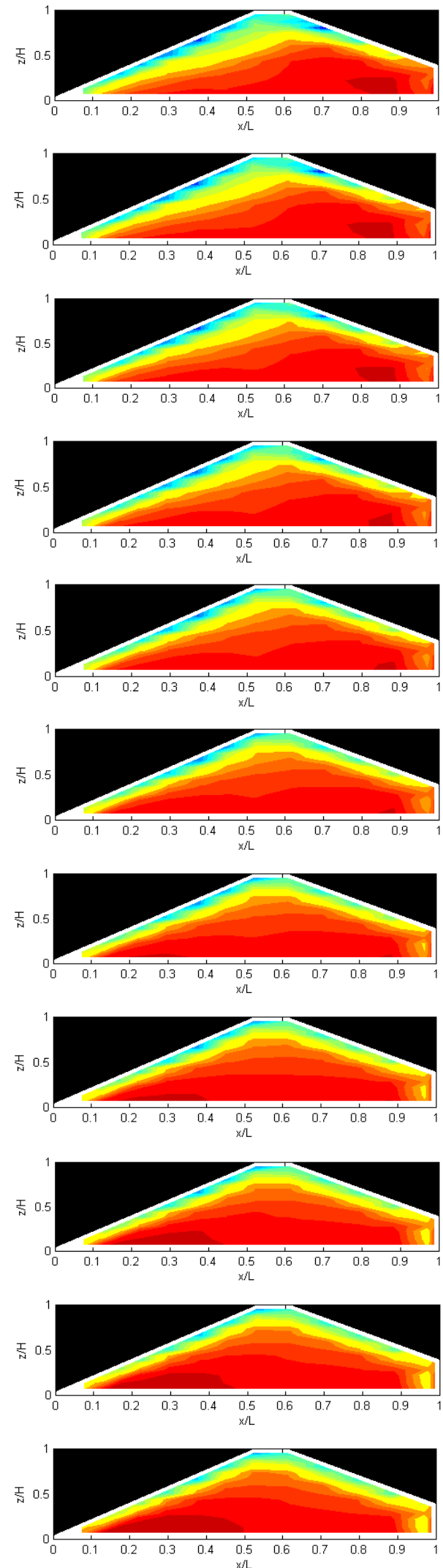